\title{
Growth and Characterisation of Ge Nanowires by Chemical Vapour Deposition
}

\author{
Chuanbo Li ${ }^{1}$, Hiroshi Mizuta ${ }^{2}$ and Shunri Oda ${ }^{1}$ \\ ${ }^{1}$ Quantum Nanoelectronics Research Center, Tokyo Institute of Technology, Tokyo, \\ ${ }^{2}$ School of Electronics and Computer Science, \\ The University of Southampton, Highfield, Southampton, \\ 1 Japan \\ ${ }^{2} U K$
}

\section{Introduction}

One-dimensional Ge/Si nanostructures have attracted much attention because of their potential applications in the design of novel electronic (Fan et al, 2006; Xiang et al, 2006; Liang et al, 2007; Hu et al, 2007), photonic (Holmes et al, 2000), battery(Chan et al 2008a; Chan et al 2008b) and sensing devices (Cui et al, 2001). For examples, due to their high mobility of electrons and holes, Ge nanowires show their promising application in highspeed field-effect transistor (Xiang et al, 2006; Liang et al, 2007). And, Ge nanowires are potentially useful for high-speed quantum computing due to the long decoherence time because of their predominance of spin-zero (Hu et al, 2007; Tyryshkin et al, 2006) nuclei and the advantage of a large excitonic Bohr radius in Ge $(24.3 \mathrm{~nm})$ allowing for quantum confinement to be observed in relatively large structures (Sun et al, 2007; Maeda et al, 1991) and at high temperatures. In order to realize these applications, controllable and high quality nanowires growths are important. Much attention has been paid to the growth of Ge nanowires(Lauhon et al, 2002; Greytak et al 2004; Kodambaka et al 2007).In most cases, the nanowires are grown in chemical vapour deposition (CVD) method visa vapor liquid solid (VLS) mechanism (He et al, 2006; Kamins et al, 2004; Fuhrmann et al, 2005; Allen et al,2008; Hannon et al, 2006), in which the formation of Ge-Au eutectic plays the most important role in the synthesis of nanowires. It has been clarified that the VLS mechanism dominates the axial growth of Ge nanowires. However, the radial growth of nanowires is easily ignored and there are only few papers to talk about it. And, the impacts of the surface conditions on the VLS (vapor-liquid-solid) synthesis of Ge nanowires are rarely discussed. Properly understanding it could help us engineer the growth of nanowires.

In this chapter, the growth of Ge nanowires via CVD method will be discussed in detail. Vapor-solid-solid (VSS) growth mechanism is supposed to dominate the nanowire growth in a radial direction, which contributes to an increase in the diameter of nanowire. After the $\mathrm{Au}$ catalysts on the tip are consumed over a long growth time, the nanowire with a rough surface will be grown due to the selective VSS radial growth. The impacts of surface condition on the growth of Ge nanowires on $\mathrm{Si}(100)$ substrate will also be observed. On the $\mathrm{SiO}_{2}$-terminated $\mathrm{Si}$ substrate, high-density $\mathrm{Ge}$ nanowires can easily be grown. However, it is more complex for the growth of Ge nanowires on H-terminated Si substrate. Due to Si 
migration and formation of a native $\mathrm{SiO}_{2}$ overlayer on the catalyst surface, it retards the growth of Ge nanowires. After removing this overlayer in the HF solution, high-density and well-ordered Ge nanowires are grown. Ge nanowires cross vertically and form two sets parallel nanowires array. It is found that nanowires grew along $<110>$ directions.

Nanowires incorporating heterostructures offer numerous potential applications in nanoelectronics and photonics devices because of their narrow diameter allowing for efficient strain relaxation and the formation of epitaxial structures from lattice-mismatched materials. In this chapter, the growth of Si-Ge and Ge-Si nanowire heterostructures will also be discussed via chemical vapour deposition. Due to the influence of interface energy, differing topographies of the heterostructures were observed. On initially grown Ge nanowires, numerous Si nanowire branches were grown near the tip due to Au migration. However, on initially grown Si nanowires, high-density Ge nanodots were observed.

Here, we will also demonstrate the well position-controllable Ge nanowires grown on the $\mathrm{SiO}_{2}$ substrate by combining top-down and bottom-up methods. To realize the practical applications of Ge nanowire on a large scale, one of the key challenges is to develop a convenient and parallel method to align bottom-up nanowires into complex patterns or structures. A "pick and place" method is most widely used for integrating nanowires. However, it lacks control of precision and repeatability, and easily induce contamination in the wires. It is expected to selectively grow nanowires directly onto desired areas of the substrate and in situ fabricate the nanowire devices. By optimizing the EBL process, $\mathrm{Au}$ patterns with a diameter of $10 \mathrm{~nm}$ were prepared by the lift-off method. The growth of Ge nanowires can be precisely controlled by adjusting the location of catalysts, which offers the possibility of in situ fabrication of nanowire devices.

\section{VSS radial growth of Ge nanowires}

All Ge nanowires were grown by using a low pressure CVD method at $300-350{ }^{\circ} \mathrm{C}$ with $10 \% \mathrm{GeH}_{4}$ precursors (in an atmosphere of hydrogen) under the total pressure of 5 torr. $\mathrm{Au}$ catalysts layers were evaporated by electron beam evaporation at room temperature on $\mathrm{SiO}_{2}$ and H-terminated substrates.
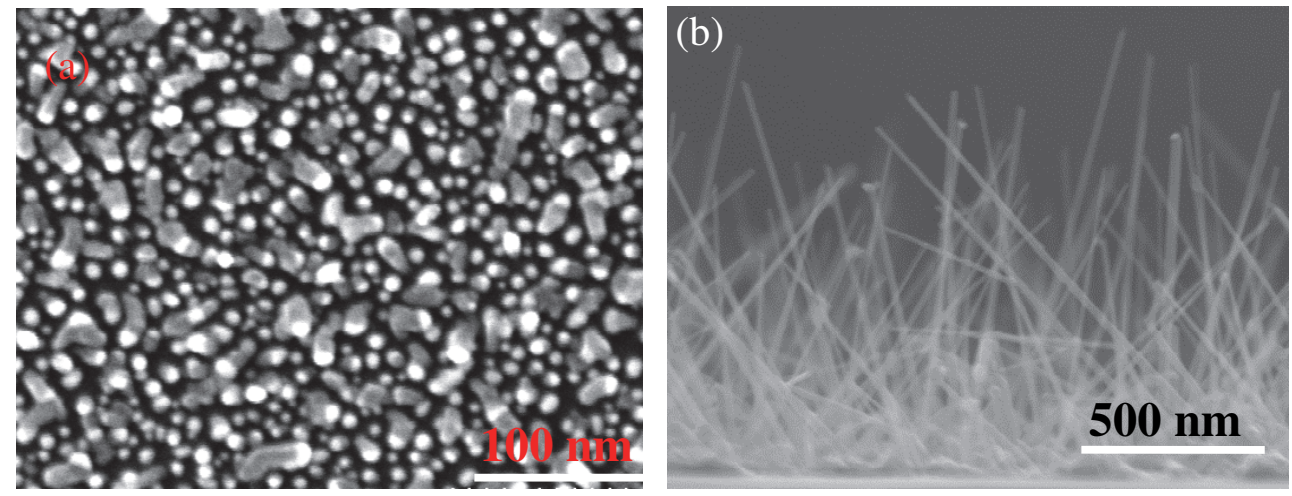

Fig. 1. (a), A SEM image of Ge nanowire at the initial growth stage grown at $300^{\circ} \mathrm{C}$; (b), A SEM image of Ge nanowires grown on the $\mathrm{SiO}_{2}$ substrate at $300^{\circ} \mathrm{C}$ for $20 \mathrm{~min}$. The nanowires have a length of $1 \mu \mathrm{m}$ and a diameter of 15 25 nm. (Li et al, 2009a ). (CAmerican Institute of Physics. 
At the initial growth stage, Ge nanowires only have a diameter of 5 10 nm, which are consistent with that of Au catalysts as deposited (Fig. 1(a)) (Li et al, 2008). However, with increasing the growth time, both the diameter and length of nanowires increase. As the scanning electron microscope (SEM) results shown in Fig. 1(b), the nanowires of 15 25 nm in diameter and $1 \mu \mathrm{m}$ in length were grown at $300{ }^{\circ} \mathrm{C}$ for $20 \mathrm{~min}$. Compared with the fast VLS growth rate in the axial direction, the growth rate in the radial direction, which contributes to an increase in the diameter of the nanowires, is much slower. There must be some other growth mechanisms instead of VLS to contribute to the nanowire growth in the radial direction. According to our control experiment results on the $\mathrm{SiO}_{2}$ substrate, the chemical vapor deposition without the assistance of $\mathrm{Au}$ catalysts can be ignored. So we believe that the vapor-solid-solid (VSS) (Kodambaka et al 2007) growth mechanism should dominate the growth of nanowires in the radial direction.

As J. B. Hannon and his coworkers (Hannon et al, 2006) investigated before, there is gold migration on the surface of nanowires during the growth of nanowires. Some Au atoms will cover, wet and smooth the nanowire sidewalls. This gold migration on the nanowire surface will consume the Au droplets on the tip of nanowires, make the droplet size smaller and induce non-uniform diameter with the thicker bottom and thinner top as shown in Fig. 1(b). Clearly, some Au catalysts exist on the Ge nanowire surface. And the Au-Ge alloy will be formed on the Ge nanowire surface at growth temperature $\left(300^{\circ} \mathrm{C}\right)$.

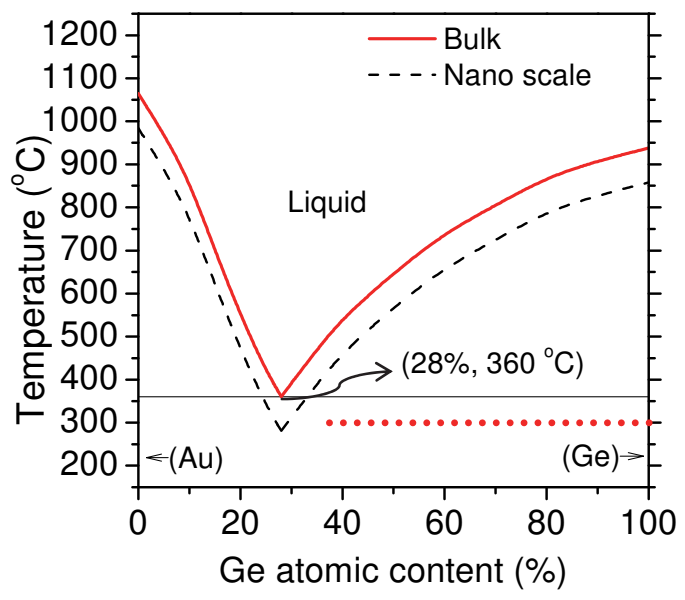

Fig. 2. A Au-Ge binary alloy diagram. Solid and dashed lines are the liquid lines for bulk material and nanoscale material respectively. Due to the size effects of nanoscale $\mathrm{Au}$ particles and nanowires, the liquid line in the binary alloy diagram has a downshift and $\mathrm{Au}-$ Ge alloy will have a lower eutectic temperature. (Li et al, 2009a). (CAmerican Institute of Physics.

However, compared with a large number of $\mathrm{Ge}$ atoms, the $\mathrm{Au}$ atoms on the surface of nanowires are much less. And the Au content in the Au-Ge binary alloy phase diagram (Kodambaka et al 2007 ) should be lower than their eutectic point $(28 \%)$. Therefore the melting point of Au-Ge alloys on the nanowire surface is higher than both their eutectic temperature and growth temperature $\left(300^{\circ} \mathrm{C}\right)$ even though the melting point of the Au-Ge 
alloy has a little downshift due to the size effect of the nanoscale Au catalyst (Adhikari et al,2006; Buffat et al,1976) as shown in Fig.2. And the Au-Ge alloy at the nanowire surface is in solid state at $300{ }^{\circ} \mathrm{C}$ located somewhere on the dotted line in the phase diagram as shown in Fig.2. So Ge atoms can only deposit on the Ge nanowire surface in the VSS mode with the assistance of $\mathrm{Au}$ catalyst to contribute to the increase of the nanowires diameter.

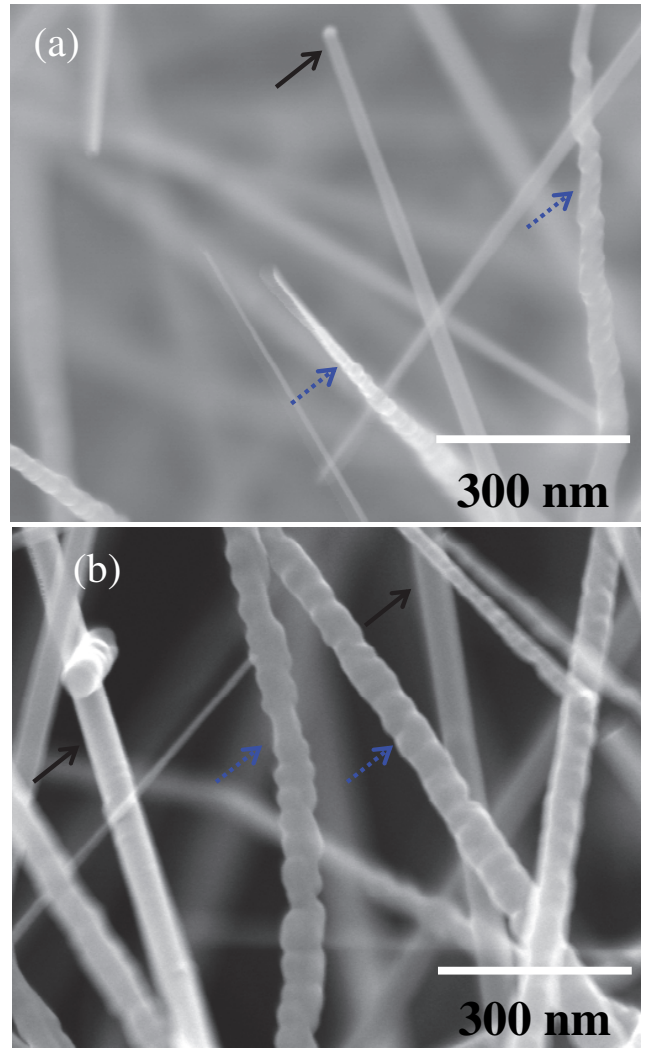

Fig. 3. SEM images of Ge nanowires grown at $300^{\circ} \mathrm{C}$ for $60 \mathrm{~min}$ on $\mathrm{SiO}_{2}$ substrate. (a) the top part and (b) the middle part of Ge nanowires; the nanowires have the length of over $3 \mu \mathrm{m}$ and the diameters of over $30 \mathrm{~nm}$. The nanowires with the Au catalyst on the tip as indicated by solid arrows exhibit a smooth surface, and the VLS axial growth is not hindered; For the nanowires without the Au catalyst on the tip as indicated by the dotted arrows, a rough surfaces are shown due to the selective VSS radial growth. (Li et al, 2009a). (c American Institute of Physics.

This hypothesis can be proven by conducting the nanowire growth for a longer period. With further increasing the growth time of Ge nanowires, the Au droplets will be consumed eventually to cover the surface of Ge nanowires, and the VLS growth in the axial direction will stop at the same time. However, the VSS growth in the radial direction does not stop yet since some Au catalysts still covers the nanowire surface. So the diameter of the nanowires still increases due to the VSS growth. At the same time, the surface area of nanowires will 
increase too, and the Au catalysts covering the nanowire surface become thinner and thinner since there is no Au atom supplied from the tip of nanowire, which migrates and covers the nanowire surface (Hannon et al, 2006). Finally, with an increase in diameter, Au catalysts cannot cover the whole nanowire surface even in an atomic layer thickness and cover only in patches. Ge atoms could be adsorbed only on the area covered with Au catalysts in the VSS mode, which contributes to the radial growth. However the radial growth does not progress in the area without the Au catalysts. So this selective VSS radial growth induces the rough nanowire surface over a longer growth time as indicated by dotted arrows shown in Fig. 3(a) and 3(b). Clearly, for the nanowire with the Au catalysts on the tip as the solid arrow indicated in Fig. 3(a), it exhibits a smooth surface because of the Au migration and wetting. And the axial growth continues. But, once the Au catalyst on the tip is consumed, due to the effect of selective VSS radial growth as mentioned above, the nanowires begin to show a rough surface and a taper shape as indicated by the dotted arrows in Fig. 3.

\section{The influences of surface conditions on the growth of germanium nanowires}

From our experimental results, we found ( $\mathrm{Li}$ et al, 2008) that the growth of Ge nanowires was quite sensitive to the surface condition of the substrate. A clear understanding of this phenomenon could help us engineer the growth of nanowires.

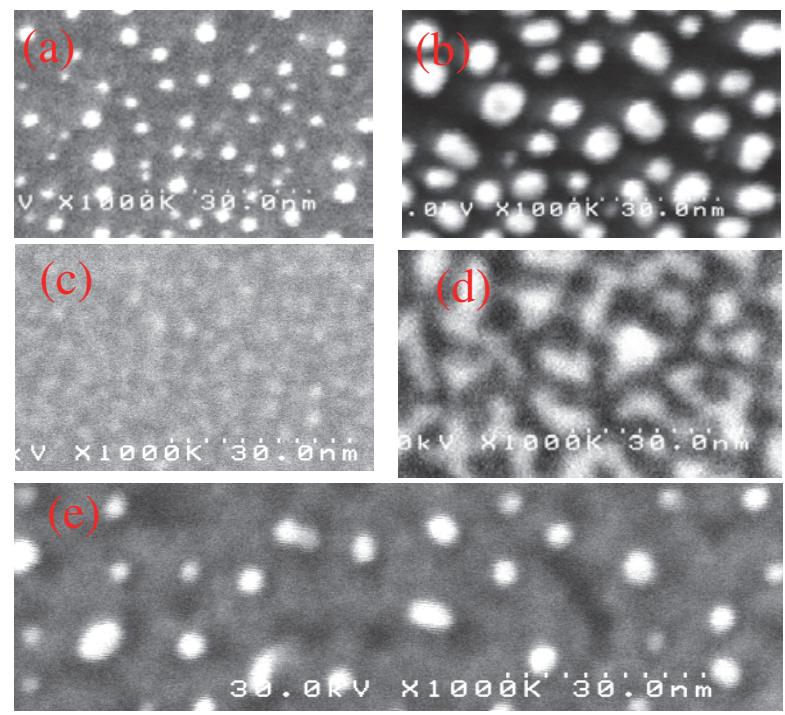

Fig. 4. SEM images of Au catalysts evaporated on $\mathrm{SiO}_{2}$-terminated $\mathrm{Si}$ substrate with a thickness of $0.1 \mathrm{~nm}$ (a) and $1 \mathrm{~nm}$ (b); Au catalysts evaporated on H-terminated Si substrate with a thickness of $0.1 \mathrm{~nm}$ (c) and $1 \mathrm{~nm}(\mathrm{~d})$; SEM image of $1 \mathrm{~nm}$-thick Au evaporated on $\mathrm{H}$ terminated Si after HF treatment for 2 minutes (e). (Li et al, 2008). (C) American Institute of Physics.

Au catalyst layers with a thickness of $0.1 \mathrm{~nm}$ and $1 \mathrm{~nm}$ were evaporated by using electron beam evaporation at room temperature on two different substrates, $\mathrm{SiO}_{2}$-terminated (a few 
nanometers native $\mathrm{SiO}_{2}$ layer or $170 \mathrm{~nm}$ thermal oxidized $\mathrm{SiO}_{2}$ ) and $\mathrm{H}$-terminated (the wafers were dipped in $1.5 \% \mathrm{HF}$ solution for 1 minute and immediately loaded in the chamber for EB evaporation) Si (100) substrates. In order to satisfy the rules of minimum surface energy, the evaporated Au layer shows different topography at different substrate surface conditions as shown in Fig. 4(a)-4(d). On the $\mathrm{SiO}_{2}$-terminated substrate, because condensed $\mathrm{Au}$ adatoms are bound more tightly to each other than to the substrate ${ }^{13}$, these atoms encounter other atoms, nucleate and agglomerate to form stable islands of 2 10 nm in diameter (Fig. 4(a), 4(b)). A thicker Au layer will result in the formation of bigger $\mathrm{Au}$ islands. On the other hand, on the H-terminated Si substrate, the Au catalyst prefers to deposit in the Stranski-Krastanov (SK) mode to form small Au islands with a wetting layer as shown in Fig.4(c) and 4(d).
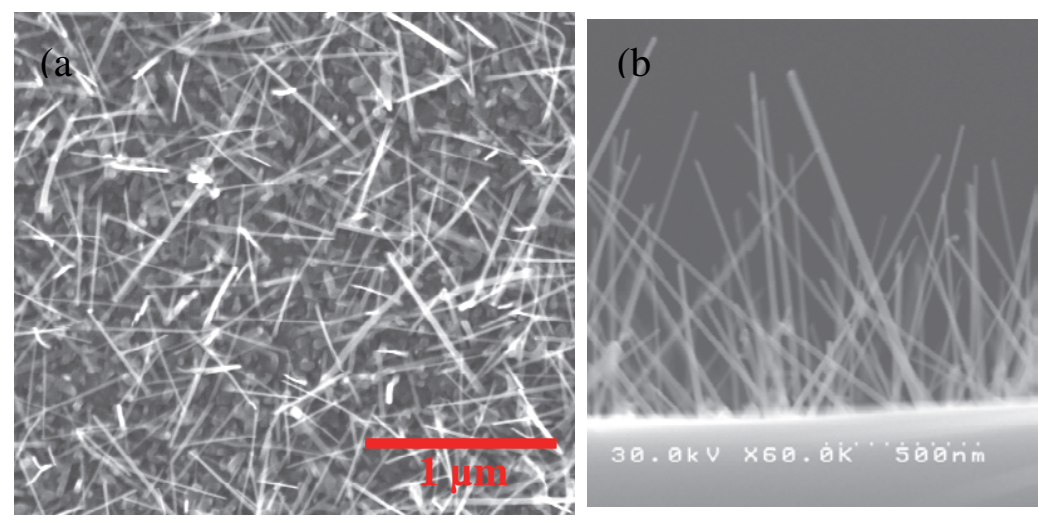

Fig. 5. (a)A top-view SEM image of high-density Ge nanowires grown on 1 nm-thick Au catalyst evaporated on the $\mathrm{SiO}_{2}$-terminated $\mathrm{Si}$ substrate. (b), a cross-sectional SEM image. (Li et al, 2008). (C) American Institute of Physics

On the $\mathrm{SiO}_{2}$-terminated $\mathrm{Si}$ substrate, without any pre-treatment to the catalysts, high-density Ge nanowires of 5 20 nm in diameter were grown on the Au catalysts with a thickness of 1 $\mathrm{nm}$ (Fig.5) and $0.1 \mathrm{~nm}$. Both high-resolution transmission electron microscopy (TEM) and Xray diffraction $(\mathrm{XRD})$ results indicate high-quality and single-crystalline Ge nanowires with the cubic diamond structure. It was found that the thickness of the $\mathrm{SiO}_{2}$ layer, either a fewnanometer-thick native oxide layer or a thicker thermally-oxidized $\mathrm{SiO}_{2}$, has no influence on the growth of Ge nanowires.

However, very few Ge nanowires were grown on the H-terminated Si substrate under the same growth conditions (Fig.6). Even after high temperature pre-annealing $\left(650{ }^{\circ} \mathrm{C}\right.$ in vacuum) to dewet the Au wetting layer, it did not have any significant influence on the growth of Ge nanowires.

While observing topography of the Au catalysts evaporated on the H-terminated substrate by SEM, we found that the contrast between Au metal and Si substrate at edge area was smaller, and the brightness of Au metal in the SEM image got weaker compared with that evaporated on the $\mathrm{SiO}_{2}$ substrate. It is likely that $\mathrm{Au}$ catalysts are covered by some insulating layers. So maybe the formation of a very thin Si oxide overlayer due to the gold catalyzed migration of Si through the gold film (Hiraki et al,1971, Lay, 1983; Hiraki 1984; Jagannathana et al 2006 ) retards the growth of Ge nanowires. 

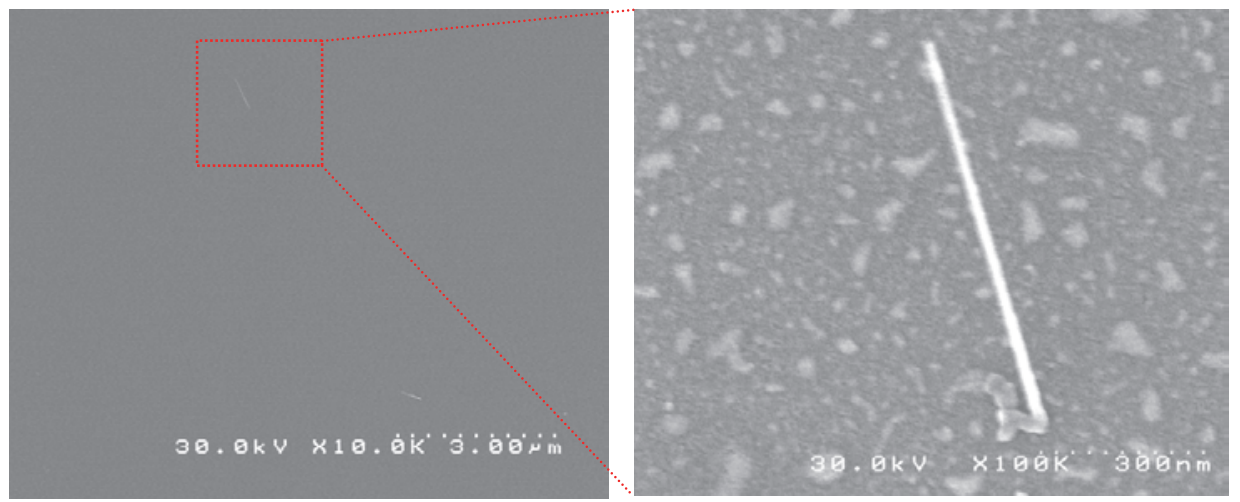

Fig. 6. A top-view SEM image of Ge nanowires grown on the H-terminated Si substrate. The inset shows a blow-up of one wire and it exhibits the same diameter as that grown on the $\mathrm{SiO}_{2}$-terminated substrate ( $\mathrm{Li}$ et al, 2008). (C) American Institute of Physics

In order to verify this hypothesis, Au catalysts evaporated on the H-terminated substrates were dipped into $1.5 \%$ HF solution for 2 minutes, and then immediately loaded into the growth chamber to grow Ge nanowires. On 1-nm-thick Au catalysts substrate, high-density and well-ordered Ge nanowires were grown as shown in Fig. 7(a). Obviously, it has a great improvement for Ge nanowires growth by removing this overlayer and it confirms that the $\mathrm{SiO}_{2}$ overlayer formed on the catalyst surface prevents the growth of Ge nanowires. Fig. 4(e) shows the SEM image of 1-nm-thick Au catalysts after HF treatment. Clear Au dots can be found after removing the $\mathrm{SiO}_{2}$ overlayer. In the case of 0.1-nm-thick Au catalysts, even after HF treatment, no nanowire was grown. We did not find any Au dots after HF treatment in the SEM image since it still remains at the wetting layer stage. So there are no nucleation centres for $\mathrm{Ge}$ atoms to grow in the axial direction. And the $\mathrm{GeH}_{4}$ precursors will be further decomposed and deposited to form a film since the AuSi eutectic alloy exits on the whole surface. Compared with the random-directed $\mathrm{Ge}$ nanowires grown on the $\mathrm{SiO}_{2}$-terminated substrate as shown in Fig.5, Ge nanowires grown on the H-terminated $\mathrm{Si}$ (100) substrate show a more ordered structure. Almost all Ge nanowires cross vertically and form two sets parallel nanowires array. It should be point out that each set of Ge nanowires has around $45^{\circ}$ angle with the [110] cleavage direction as labelled in Fig. 7(a). Till now, only two growth directions, [110] and [111] were found for Ge nanowires grown on the Si substrate. Based on this finding and our experiment results, we believe that Ge nanowires are grown preferably in the [101], [011], [-101] and [0-11] directions as the four dashed arrows shown in Fig. 7(b), in which these four growth directions are crossed vertically and have a $45^{\circ}$ degree with [110] and [-110] cleavage directions. It agrees well with the experiment results as shown in Fig. 7(a). The two dashed-dotted arrows in Fig. 7(b) are [110] and [-110] cleavage directions for (100) wafer as labelled in Fig. 7(a).

During the growth of Ge nanowires on Au catalysts evaporated on the H-terminated Si substrate after HF treatment, $\mathrm{GeH}_{4}$ will decompose in the AuSi eutectic alloy located at the interface between the single-crystalline Si surface and Au layer and then supersaturated Ge atoms will separate out to grow in the axial direction. This ordered structure at the interface can induce the Ge nanowires grow in only the $<110>$ directions. This capability to control the nanowire growth direction would be very important to engineer the transport 
characteristics for electrons and holes individually to obtain the best switching performance of a complementary logic circuit configuration ${ }^{19}$. And also it is quite attractive for future large-scale nanowire integration.
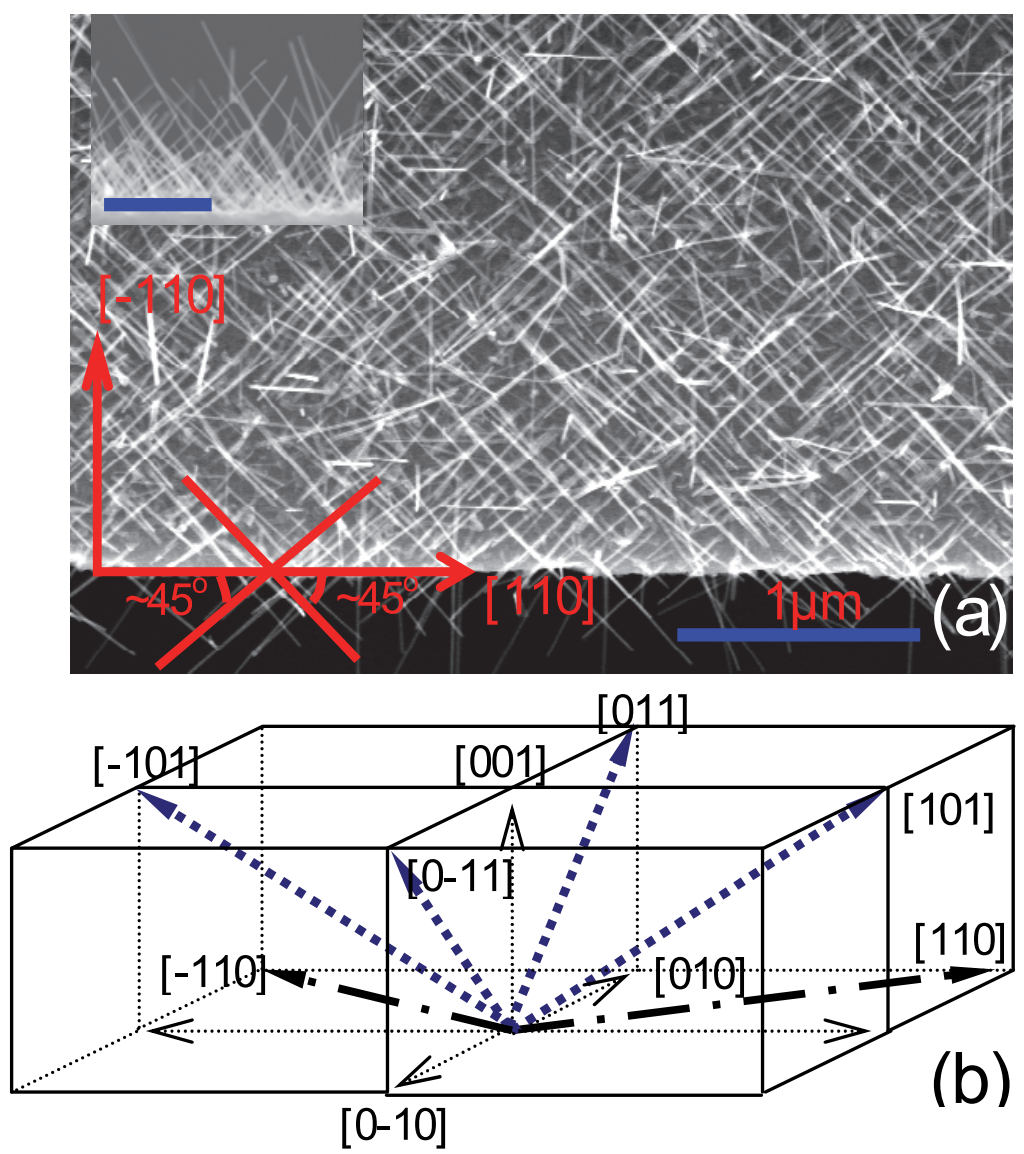

Fig. 7. (a), A top-view SEM image of high density and well-ordered Ge nanowires grown on 1-nm-thick Au catalysts evaporated on the H-terminated Si (100) after dipped into the HF solution to remove the $\mathrm{SiO}_{2}$ overlayer. The bottom edge is the [110] cleavage direction. The inset shows its side-view SEM image and the scale bar is $500 \mathrm{~nm}$; (b), A 3D schematic of the growth directions of Ge nanowires on $\mathrm{Si}$ (100) wafer. Four dashed arrows directed to [101], [011], [-101] and [0-11] are the Ge nanowires growth directions. (Li et al, 2008). (C) American Institute of Physics

In the case of $\mathrm{Au}$ catalysts evaporated on the $\mathrm{SiO}_{2}$-terminated substrate, strong bonding between $\mathrm{Si}$ and $\mathrm{O}$ atoms may prevent the migration of $\mathrm{Si}$ atoms to the surface and the formation the $\mathrm{SiO}_{2}$ overlayer. Therefore $\mathrm{Ge}$ nanowires can be grown without $\mathrm{HF}$ treatment.

Figures 8(a) and 8(b) show the SEM images of Ge nanowires grown at $350{ }^{\circ} \mathrm{C}$ in the same conditions on the $\mathrm{H}$-terminated $\mathrm{Si}$ and the $\mathrm{SiO}_{2}$-terminated $\mathrm{Si}$ substrates, respectively. The 
nanowires showed different density, shape and size on these two substrates. On the Hterminated substrate, similarly with that grown at $300{ }^{\circ} \mathrm{C}$ as discussed above, only lowdensity $\mathrm{Ge}$ nanowires can be grown because the $\mathrm{SiO}_{2}$ overlayer covering the Au catalysts surface hinders the growth of Ge nanowire,. At high temperature, the growth speed increases, and thicker and longer nanowires can be grown. However, on the $\mathrm{SiO}_{2-}$ terminated substrate, thicker and shorter nanowires were grown. Compared with longer nanowires with a smooth surface as shown in Fig. 8(a), the difference between these nanowires should result from the different Au atoms supply. In the case of low-density Ge nanowires growth, there is enough $\mathrm{Au}$ atoms supply, which migrate from the other areas and contribute to the VLS growth over a longer period. However, on the $\mathrm{SiO}_{2}$-terminated substrate, the Au catalyst droplets will supply for more nanowires growth and are easily consumed. After there remains no Au supply for the axial direction growth, thicker nanowires with a rough surface are grown. These facts evidence the above-mentioned explanation.

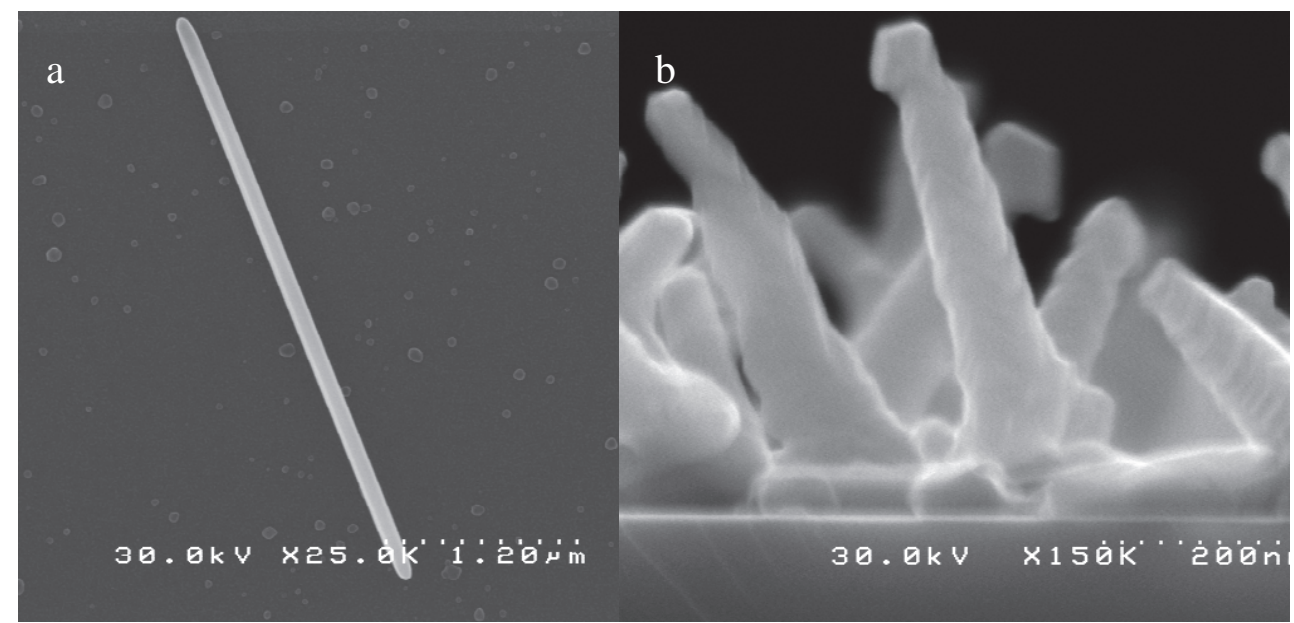

Fig. 8. SEM images of Ge nanowires grown at $350^{\circ} \mathrm{C}$ for 20 minutes on H-terminated (a) and $\mathrm{SiO}_{2}$-terminated (b) $\mathrm{Si}$ substrate, respectively.

\section{Growth of Ge-Si nanowire heterostructures}

Nanowire heterostructures offer numerous potential applications in nanoelectronics and photonics devices because their small diameter enables efficient strain relaxation and the formation of epitaxial structures from lattice-mismatched materials (Dick et al, 2007a; Ertekin et al, 2005; Kastnery \& Gosele 2004; Gudiksen et al, 2002). Enormous effort has been made to the growth of Ge-Si nanowies due to their low cost and unrivaled compatibilities with the main-stream ultra-large-scale Si integration technology ( $\mathrm{Lu}$ et al 2005; Liang et al 2007; $\mathrm{Hu}$ et al, 2007; $\mathrm{Hu}$ et al 2008). Some heterostuctures, such as $\mathrm{Ge} / \mathrm{Si}$ core/shell nanowires (Xiang et al, 2006;Lauhon et al, 2002; Ben \& Patolsky, 2010)and Si/SiGe nanowires superlattice (Wu et al, 2002; Clark et al, 2008) have been grown via the VLS mechanism. 

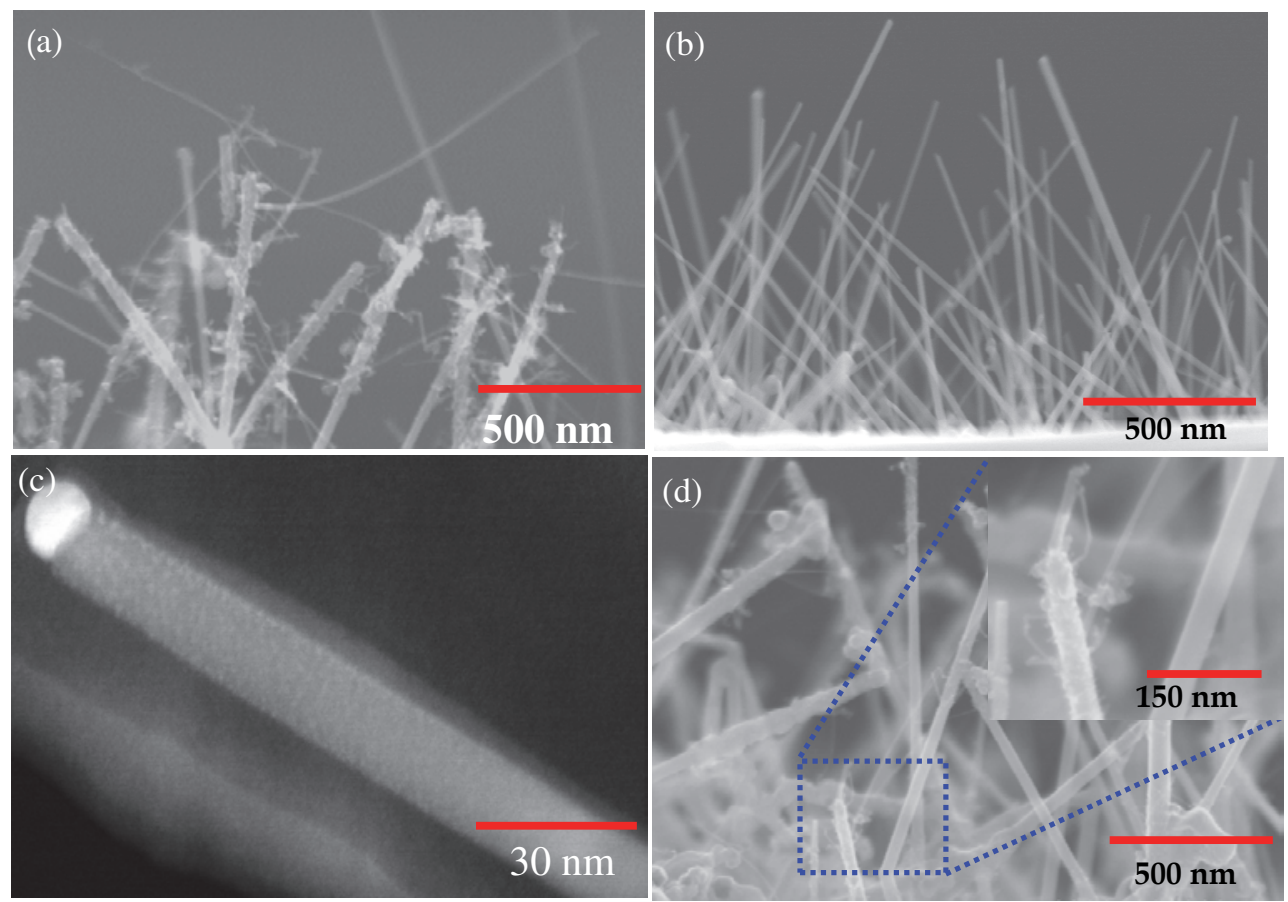

Fig. 9. (a), A SEM image of branched Si/Ge nanowire heterosturctures grown at $350^{\circ} \mathrm{C}$ on Ge nanowire substrate in the continuous mode (top part of the nanowires); (b), A Crosssectional SEM image of $\mathrm{Ge}$ nanowires grown on $\mathrm{SiO}_{2}$ substrate at $300^{\circ} \mathrm{C}$ for $20 \mathrm{~min}$; (c), A dark field TEM image of Ge nanowires before the growth of Si nanowire branches. The Au catalysts locate at the tips of nanowires and many small Au particles on the top parts of nanowires can be observed; (d), A SEM image of bottom part of Ge nanowires and inset shows the enlarged SEM image in the dotted square. (Li et al, 2011). (C) Elsevier.

Branched nanowires offer another approach to increase structural complexity thus enabling greater functionalities (Wang et al, 2004). They lead to more potential applications in nanoelectronic devices by increasing the number of connection points and providing a means for parallel connectivity and interconnection of functional elements (Dick et al, 2007b). Moreover, hierarchically heterobranching and hyperbranching nanowire structures will potentially promote applications in solar energy harvesting, sensor and so forth (Bierman \& Jin, 2009). A clear understanding of the growth mechanism of branched nanowires would enable to control and design the complex heterostructures which meet specific requirements of challenging applications. The growth of homogenous $\mathrm{Si}-\mathrm{Si}$ nanowire branches has been demonstrated to date (Wang et al, 2004; Doerk et al, 2008). Here the growth of $\mathrm{Si}-\mathrm{Ge}$ and Ge-Si nanowire heterostructures is also presented.

Si nanowires were grown at $350^{\circ} \mathrm{C}$ for $20 \mathrm{~min}$ by using the $\mathrm{Si}_{2} \mathrm{H}_{6}(3.3 \mathrm{sccm})$ and $\mathrm{H}_{2}(50 \mathrm{sccm})$ at 1 Torr. There is a $40 \mathrm{~min}$ waiting time to stabilize the growth temperature in a vacuum condition by heating (from $300^{\circ} \mathrm{C}$ to $350{ }^{\circ} \mathrm{C}$ ) or cooling $\left(350^{\circ} \mathrm{C}\right.$ to $300{ }^{\circ} \mathrm{C}$ ) the growth chamber between the growth intervals. Fig. 9(a) shows the SEM image of Ge-Si nanowire 
heterojunctions grown in a continuous mode. Compared with the smooth surface of Ge nanowire as the SEM image shown in Fig. 9(b), many nanowire branches were grown on the surfaces of Ge nanowire stems. It was confirmed that the branches on Ge nanowire stems were Si nanowires by the small angle XRD spectrum as shown in Fig. 10. Compared with the XRD spectrum of Ge nanowires without any branches (dotted line), an extra set of peaks were observed in the XRD spectrum of branched Ge nanowires (solid line). The simulation results by Philips X'pert high-score software are shown in table 1 . Clearly, the peaks at $27.4112^{\circ}, 45.3028 \circ, 53.7354^{\circ}, 65.9223 \circ, 72.8923 \circ$ and $83.8253 \circ$ match well for both samples and are related to Ge (111), (220), (311), (400), (331) and (422) plane respectively of Ge nanowires. The other set of peaks at $28.4247^{\circ}, 47.2729^{\circ}$ and $56.1197^{\circ}$ originate from the Si (111), (220) and (311) planes respectively.

\begin{tabular}{|c|c|c|c|c|}
\hline $\begin{array}{c}\text { Position } \\
\left({ }^{\circ} 2 \Theta\right)\end{array}$ & $\begin{array}{c}\text { FWHM } \\
\left({ }^{\circ} 2 \Theta\right)\end{array}$ & $\begin{array}{c}\text { d-spacing } \\
(\AA)\end{array}$ & $\begin{array}{c}\text { Relativeintensity } \\
(\%)\end{array}$ & $\begin{array}{c}\text { Chemical } \\
\text { Formula }\end{array}$ \\
\hline 27.5006 & 0.1574 & 3.24344 & 100.00 & $\mathrm{Ge}$ \\
\hline 28.4247 & 0.3936 & 3.14007 & 22.97 & $\mathrm{Si}$ \\
\hline 45.5304 & 0.1574 & 1.99231 & 51.46 & $\mathrm{Ge}$ \\
\hline 47.2729 & 0.5510 & 1.92286 & 8.86 & $\mathrm{Si}$ \\
\hline 53.8240 & 0.2362 & 1.70327 & 27.82 & $\mathrm{Ge}$ \\
\hline 56.1197 & 0.4723 & 1.63892 & 3.25 & $\mathrm{Si}$ \\
\hline 66.1912 & 0.6298 & 1.41187 & 4.03 & $\mathrm{Ge}$ \\
\hline 72.9023 & 0.5510 & 1.29757 & 7.45 & $\mathrm{Ge}$ \\
\hline 83.9378 & 0.5760 & 1.15189 & 7.19 & $\mathrm{Ge}$ \\
\hline
\end{tabular}

Table 1. Peak lists of solid line in Fig. 10 and its analysis results (Li et al, 2011). (C Elsevier.

It is interesting that the Si nanowire branches were grown on the Ge nanowire surface rather than on their tips. Since the VLS nanowire growth is sensitive to Au catalysts, there must be some Au catalysts on the surface of Ge nanowires to contribute to the growth of Si nanowire branches. It has been reported that there is an Au migration on the nanowire surface during their growth (Kodambaka et al, 2007; Hannon et al, 2006; Gosele, 2006) and during the hightemperature growth interval (Doerk et al, 2008). From the dark field transmission electron microscope (TEM) image of Ge nanowires as shown in Fig. 9(c), a rough surface with numerous white spots was observed on the top parts of Ge nanowires. It is clear that the big white spot located at the tip of nanowire is the Au catalyst. The energy-dispersivespectrometry analysis indicates that the small white dots on the top parts of nanowires are the surface-migrated $\mathrm{Au}$ particles. The density and the size of $\mathrm{Au}$ particles reduce with increase of distance from the tips of Ge nanowires and no Au particle is found on the bottom of the nanowires. This distribution of Au particles agrees well with that of $\mathrm{Si}$ nanowire branches grown on the Ge nanowire surface as SEM image shown in Fig. 9(d). 


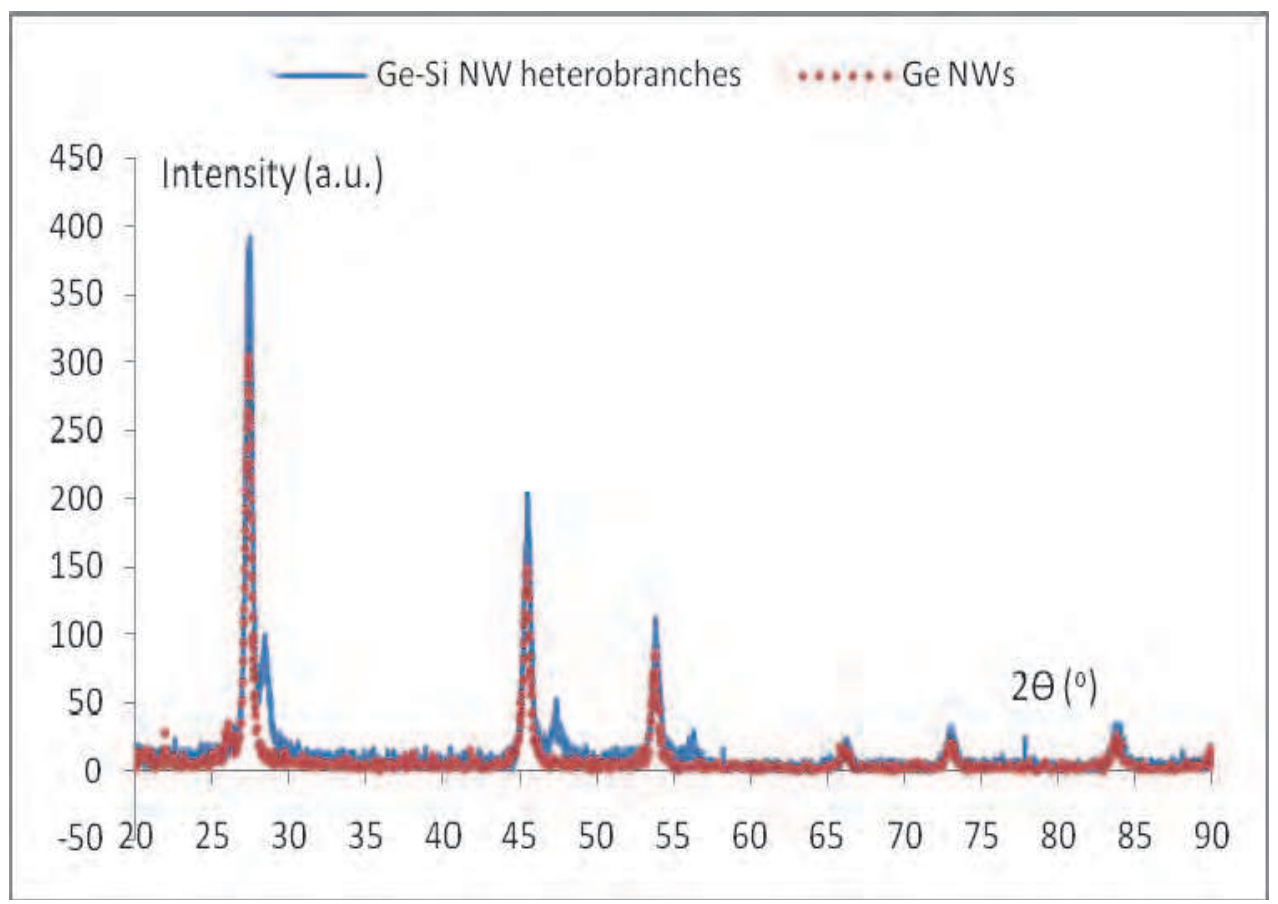

Fig. 10. Small angle XRD spectrum of Ge nanowires with and without branches. (Li et al, 2011). (C) Elsevier.

Clearly, these self-seeded Au particles formed by the surface migration, play an important role in controlling the growth of Si nanowire branches. The branches were only grown on the top parts of nanowire stems. With increasing distance from the tips of nanowires, the less Au catalyst exists on the surface, resulting in fewer Si nanowire branches due to the less nuclear centers for their growth. The enlarged SEM image in the inset of Fig. 9(d) shows a typical example where the branches only appear on the top parts of stems. On the short Ge nanowire stems, many Si branches were grown on its top. However, on the surface of its neighbors, as the bottom parts of long nanowires, no Si branch was found. The growth rate of nanowire branches is lower than that grown on planar substrate. Shorter and thinner nanowire branches were grown at the same condition due to the shortage of Au catalysts.

The experiments indicate that the growth mode has an important influence on the growth of nanowire branches. If Ge nanowires were kept in the air for a few days and then were reloaded into the chamber for $\mathrm{Si}$ nanowire branch growing in a separate mode, the $\mathrm{Si}$ heterostructures tend to only grow on the tips of Ge nanowires as shown in Fig. 11. The growth rate of $\mathrm{Si}$ nanowire branches greatly reduced compared with that grown in the continuous mode as shown in Fig. 9(a). The longer the Ge nanowires were put in air, the fewer Si nanowire branches were grown (Fig. 11(a) and 11(b)). We believe that $\mathrm{GeO}_{2}$ formed on the nanowire surface due to the oxidization of Ge atom migrated to the surface covers the small $\mathrm{Au}$ catalysts dots on the top parts of Ge nanowire and retards the growth of $\mathrm{Si}$ nanowire branches. 


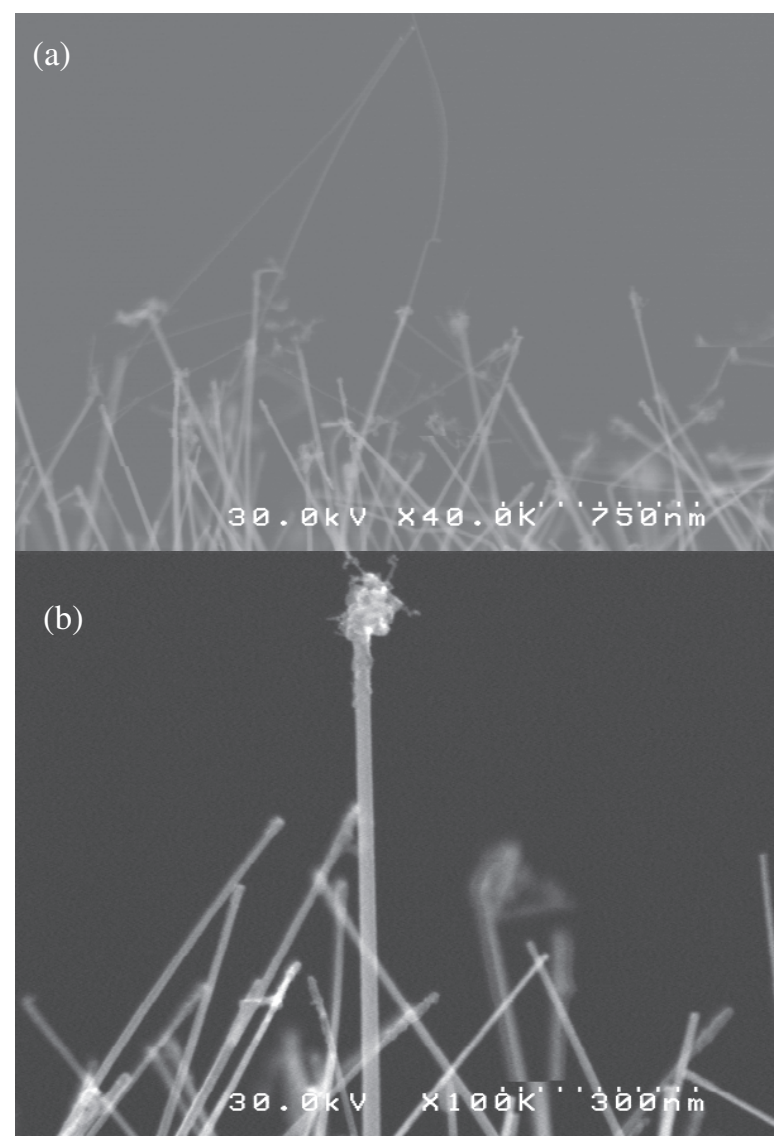

Fig. 11. SEM images of Si nanowire branches grown at $350^{\circ} \mathrm{C}$ on Ge nanowire substrates in the separate mode. Ge nanowires were firstly grown at $300^{\circ} \mathrm{C}$ and were put in air for one week (a) and three weeks (b) and then were reloaded in the chamber for growth of $\mathrm{Si}$ nanowire branches. (Li et al, 2011). (c) Elsevier.

As a comparison, Ge heterostructures on Si nanowire stems were also observed. After the synthesis of $\mathrm{Si}$ nanowires at $350{ }^{\circ} \mathrm{C}$, the chamber was cooled down to $300{ }^{\circ} \mathrm{C}$ in $40 \mathrm{~min}$, and then $\mathrm{GeH}_{4}$ was introduced into the growth chamber to grow $\mathrm{Ge}$ heterostructures. Interestingly, a different topography of heterostructures was exhibited. As shown in Fig. 12(a), Si nanowire stems have a clean surface before the growth of Ge heterostructure. However, many Ge nanodots instead of branches were observed on the surface of $\mathrm{Si}$ nanowire stems as shown in Fig. 12(b) and 12(c). K. A. Dick and his coworkers (Dick et al, 2007a) have discussed that different morphologies of crawl or nanowire heterosturctures were grown in compound nanowire systems depending on the growth condition and interface energy between these materials. Similar effects were observed in our experiment. In order to satisfy the minimum system energy, high density Ge quantum dots other than branches were grown on the surface of Si nanowire stems. 

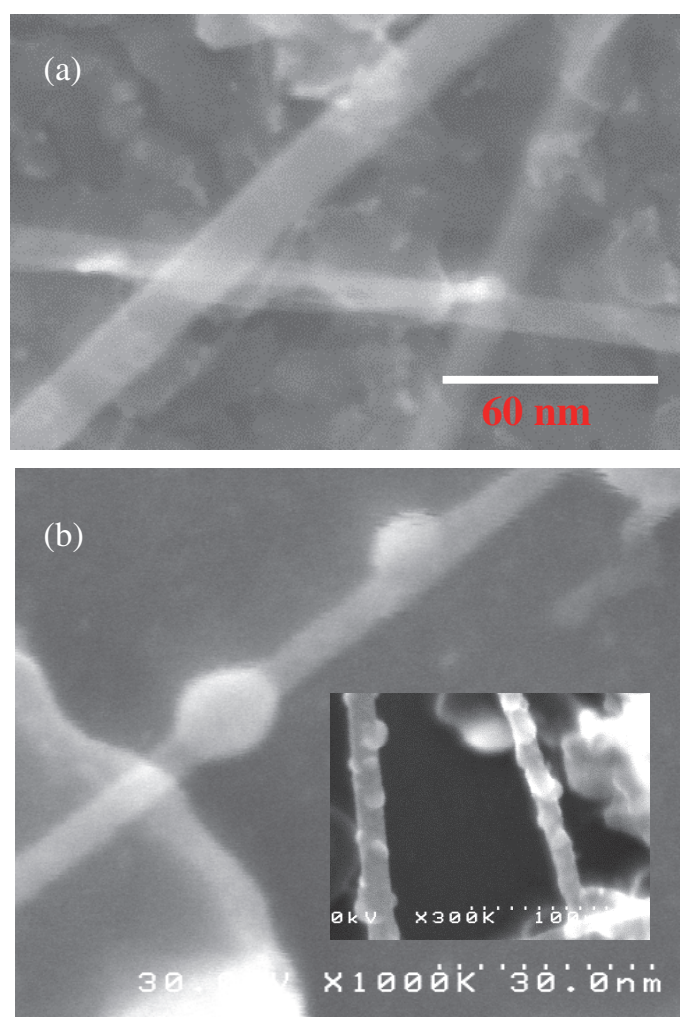

Fig. 12. (a), SEM image of $\mathrm{Si}$ nanowires grown on $\mathrm{SiO}_{2}$ substrate at $350^{\circ} \mathrm{C}$ for $20 \mathrm{~min}$; (b) and (c), SEM images of Ge nanodots heterostructure grown at $300^{\circ} \mathrm{C}$ on Si nanowire substrate in the continuous mode. (Li et al, 2011). (C) Elsevier.

At the same condition, the chemical vapor deposition of $\mathrm{Ge}$ atom without the assistance of $\mathrm{Au}$ catalysts can be ignored at $300{ }^{\circ} \mathrm{C}$ according to our control experiment results on $\mathrm{SiO}_{2}$ substrate. The nanodot heterostructures are also catalyzed by the $\mathrm{Au}$ catalyst on the $\mathrm{Si}$ nanowire surface. The Ge nanodots have a diameter of 2-10 nm, which is much smaller than those grown on the Si substrate in the Stranski-Krastanov mode (Li et al, 2004a; Li et al, $2004 \mathrm{~b}$ ). These small size nanodot herterostructures will have much potential application when applied in quantum computation and optical communication.

\section{Position-controllable single Ge nanowire array}

To realize the applications of Ge nanowires on a large scale, one of the key challenges is to develop a convenient and parallel method to align the bottom-up nanowires into the complex patterns or structures ( $\mathrm{Li}$ et al, 2007). Recently, a "pick and place" method is most widely used for the integrations of nanowires. However, these processes lack control of in precision and repeatability, and easily induce contamination in the wires (Conley et al, 2005). It is expected to selectively grow nanowires directly onto the desired areas of the 
substrate and in situ fabricate the nanowire devices (Li et al, 2004c). In the vapor-liquid-solid chemical vapor deposition (CVD) process, gold catalysts initiate and guide the growth of nanowires (He et al, 2006; Kamins et al, 2004; Wu et al, 2002; Kodambaka et al, 2007). Hence, precise control the location of nanowires relies on the capability to control the location of $\mathrm{Au}$ clusters (Lombardi et al, 2006; Fuhrmann et al, 2005). In this book, we also demonstrate the well position-controllable $\mathrm{Ge}$ nanowires growth on $\mathrm{SiO}_{2}$ substrate by combining top-down and bottom-up methods.

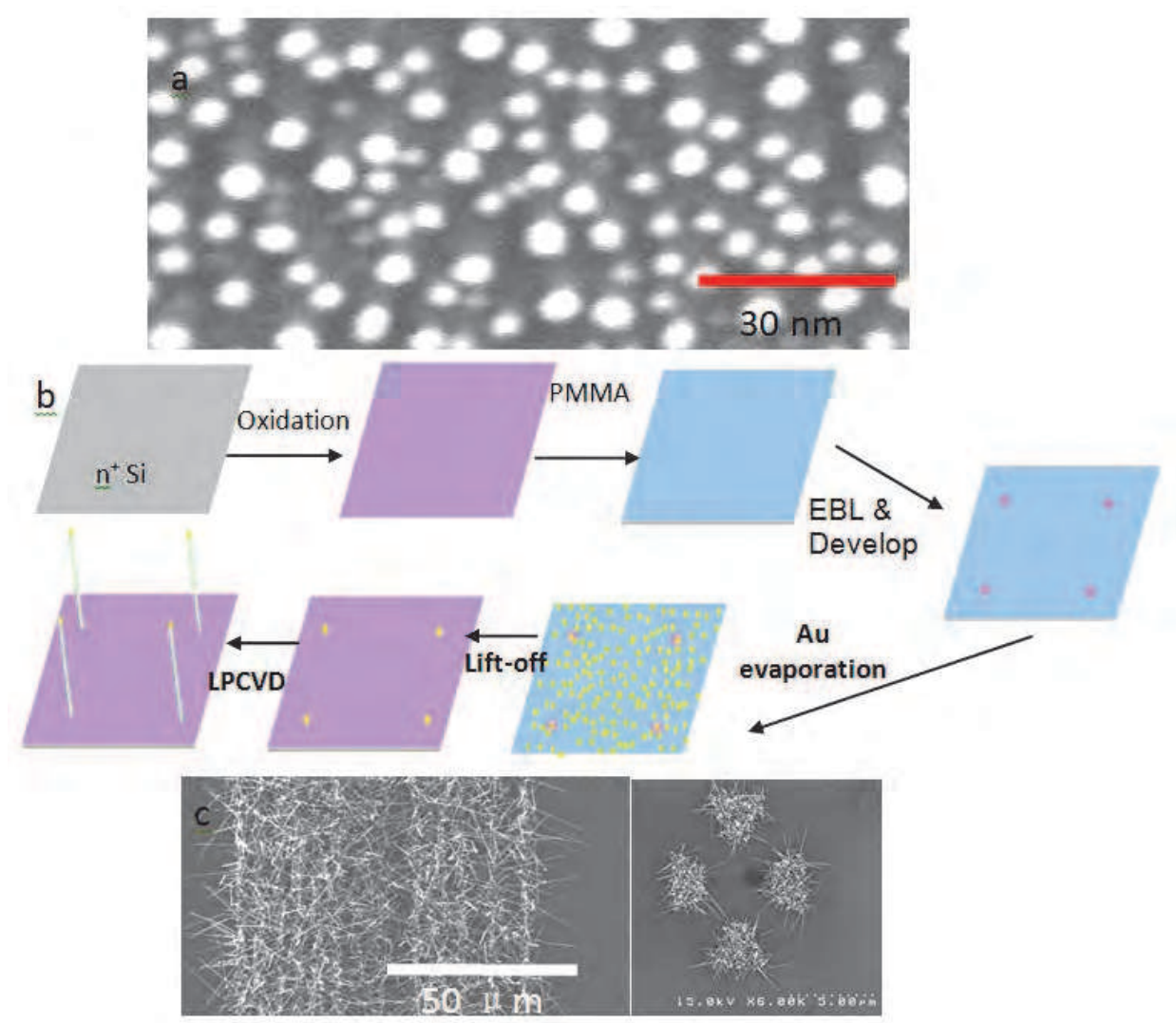

Fig. 13. (a), A SEM image of Au catalysts evaporated on $\mathrm{SiO}_{2}$ substrate. The Au dots have a diameter of around 3 8 nm; (b), The schematic of process for the patterning Au catalysts and the growth of Ge nanowires; (c), SEM images of Ge nanowires patterns grown on the $\mathrm{Au}$ catalysts patterns. The wires are selectively grown on the areas with Au catalysts. (Li et al, 2009b). (C) Japan Society of Applied Physics.

Due to the stronger bond between the condensing $\mathrm{Au}$ adatoms than to the substrate (Venables, 1986), the $\mathrm{Au}$ atoms agglomerate to form the high-density islands with the diameter of $3 \sim 8 \mathrm{~nm}$ as shown in Fig. 13(a). The well-ordered Au catalysts were prepared by using a JEOL JBX-5FE electron-beam lithography (EBL) system with a beam voltage of $50 \mathrm{kV}$ and a beam diameter of $8 \mathrm{~nm}$ and a lift-off method. 

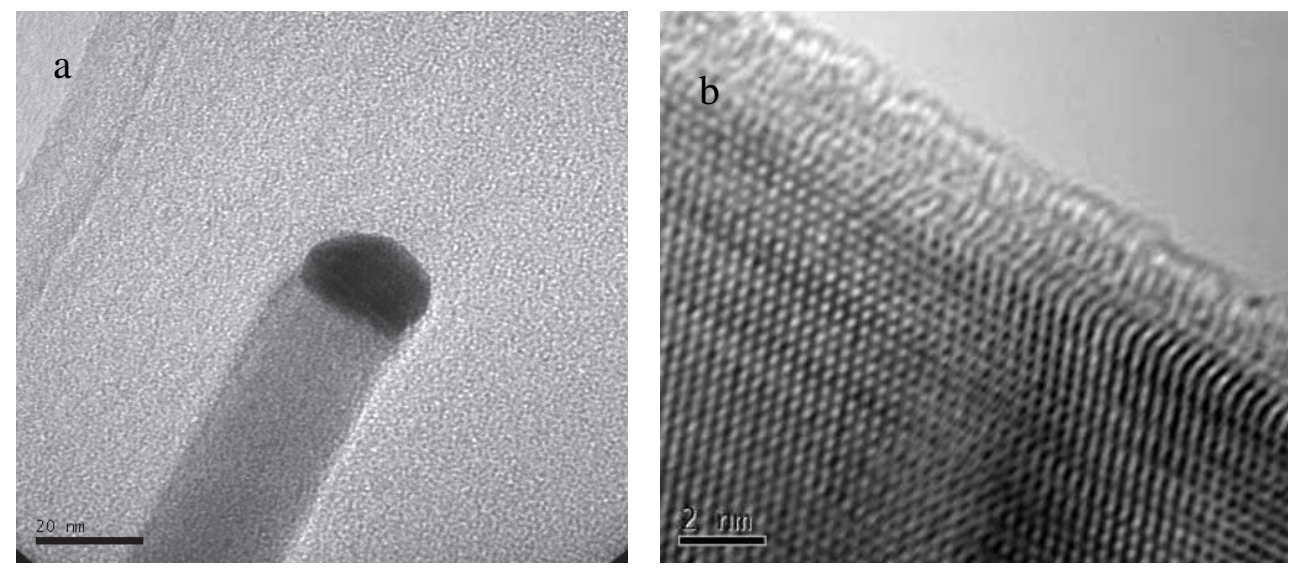

Fig. 14. (a), The TEM of Ge nanowires grown at $300{ }^{\circ} \mathrm{C}$. The dark part on the tip of the nanowire is the Au catalyst; (b), the High-resolution TEM image of Ge nanowires showing the single-crystal structure; (Li et al, 2009b). (C) Japan Society of Applied Physics.
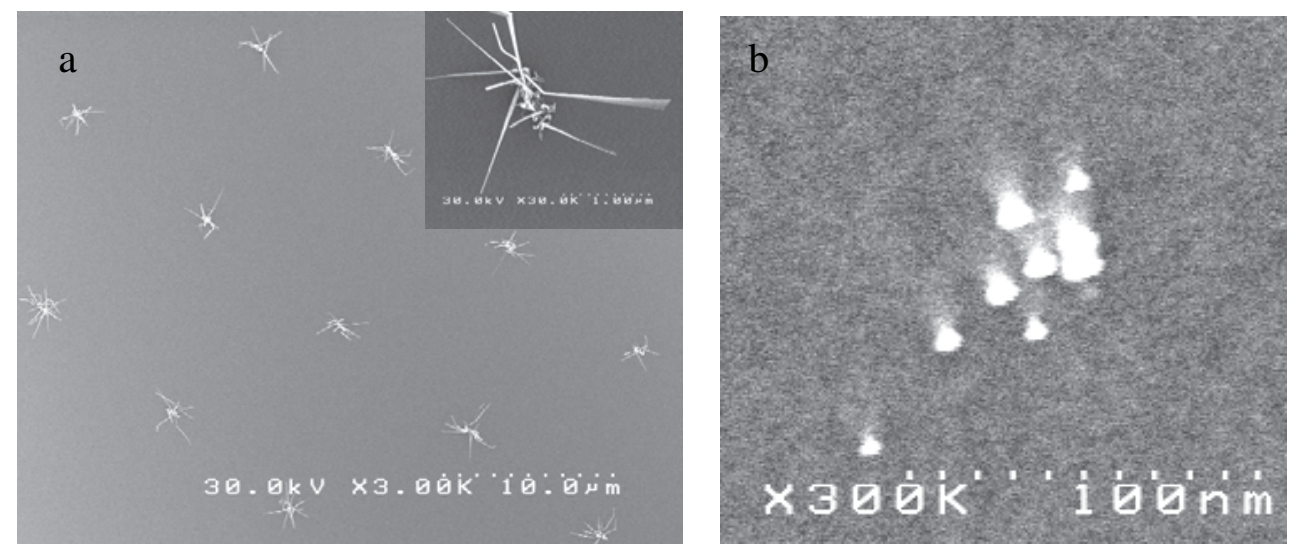

Fig. 15. (a), the SEM images of Ge nanowires array with a space distance of $10 \mu \mathrm{m}$; The inset is the enlarged image of one pattern of Ge nanowires. The scale bar in the inset is $1 \mu \mathrm{m}$. And (b) shows one of the Au patterns containing several Au dots. The scale bar in the inset is 100 nm. (Li et al, 2009b). (C) Japan Society of Applied Physics.

The schematic of the process for patterning the Au catalysts and the growth of Ge nanowires is shown in Fig. 13(b). After the $n^{+} \operatorname{Si}(111)$ wafer was oxidized at $1100{ }^{\circ} \mathrm{C}$ for 1 hour, the positive-type diluted (50\%) polymethyl methacrylate (PMMA) EB resist with a thickness of $40 \mathrm{~nm}$ was coated at $8000 \mathrm{rpm}$ and was prebaked at $170{ }^{\circ} \mathrm{C}$ for 30 minutes. Then, the sample was exposed using the EBL under a dose condition of $400 \mu \mathrm{C} / \mathrm{cm}^{2}$ and were developed by xylene for 60 seconds followed by a rinse with iso-propanol for 30 seconds. After the evaporation of Au particles and lift-off, Au pattern was formed. Finally, Ge nanowires were grown on the Au pattern by LPCVD. 

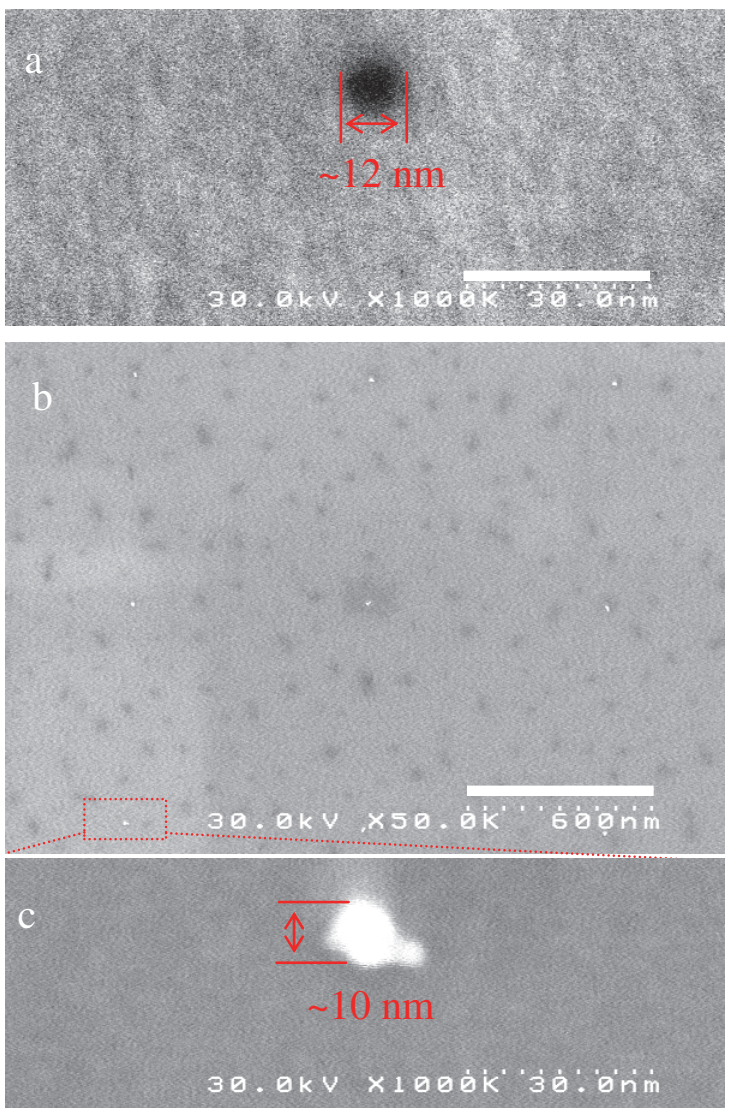

Fig. 16. (a), SEM image of one hole formed by EBL. The black hole area with a diameter of 12 $\mathrm{nm}$ is the $\mathrm{SiO}_{2}$ substrate and the gray area is PMMA layer; (b), SEM image of the wellordered Au dots array with a space of $700 \mathrm{~nm}$. The white dots in the image are Au catalysts; and (c), the enlarged image of one of the Au dots in fig. 16(b). (Li et al, 2009b). (C) Japan Society of Applied Physics.

Fig. 13(c) shows a SEM image of high-density Ge nanowire strips grown on patterned $\mathrm{Au}$ catalysts. Ge nanowires with a diameter of $5 \sim 20 \mathrm{~nm}$ can be grown selectively on the area with Au catalysts. A TEM of Ge nanowires is shown in Fig. 14(a). Almost all of the nanowires are defect free over the whole length that could be observed. The dark parts on the tips of nanowires in Fig. 14(a) are Au catalysts. The high-resolution TEM image in Fig. 14(b) reveal the high-quality single-crystalline Ge nanowires with a lattice constant of 0.565 $\mathrm{nm}$, which is in excellent agreement with the diamond crystal structure known for Ge. The X-ray diffraction result (dotted line in Fig. 10) also indicates that the crystal structure of grown Ge nanowires is cubic diamond structure according to the typical six peaks. And two peaks related to the Au FCC structure were also found.

By reducing the size of each Au pattern to contain only a few Au particles (Fig. 15(b)), the Ge nanowire array with several nanowires in each area was obtained (Fig. 15). The inset of 
Fig. 15 shows the enlarged image of one of the patterns. Around 10 nanowires were grown in each area.

In order to realize the alignment of single Ge nanowire, the Au catalyst array with only one $\mathrm{Au}$ dot in each area is needed. One way to settle this problem is to reduce the size of pattern. In the lift-off process, the thickness of the resist plays an important role in controlling the size and shape of the design. The thinner the resist is, the easier it is to control the size of pattern. In the case of Au film with only a thickness of about $0.5 \mathrm{~nm}$, very thin resist can be applied. By reducing the PMMA resist down to $40 \mathrm{~nm}$ and optimizing the relationship between the electron dose and develop time in EBL process, a very small hole with the diameter of around $12 \mathrm{~nm}$ can be obtained as shown in Fig. 16(a), which is almost the same size as the beam spot of EBL. In Fig. 16(a), the black hole is the $\mathrm{SiO}_{2}$ substrate and the gray area is PMMA. By choosing a suitable density of the Au particles to make sure that one $\mathrm{Au}$ dot could be deposited in this kind of hole, the well-ordered single Au dot array with a space of $700 \mathrm{~nm}$ was prepared as shown in Fig. 16(b). According to the enlarged image of one pattern as shown in Fig. 16(c) we can find each Au dot has a diameter of around $10 \mathrm{~nm}$.

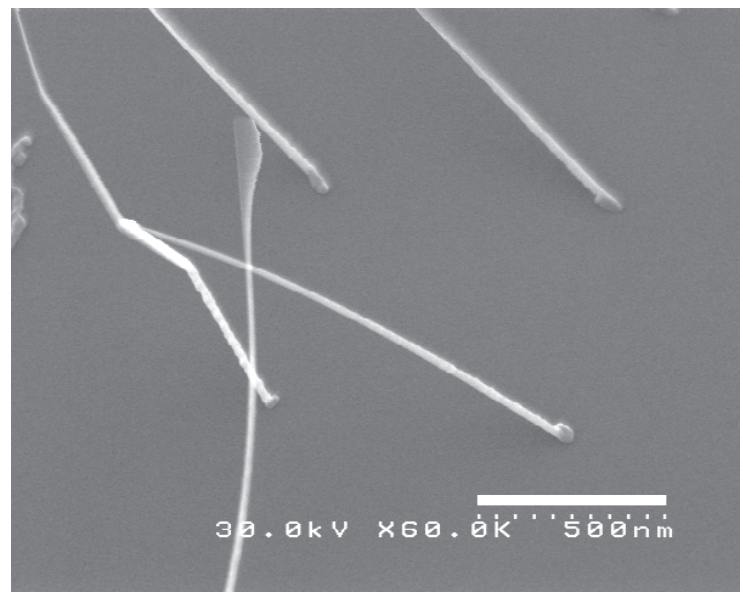

Fig. 17. A SEM image of single Ge nanowire array grown on the well-ordered Au pattern. (Li et al, 2009b). (C) Japan Society of Applied Physics.

On such well-ordered Au catalyst array, the single Ge nanowire arrays with a space of 700 $\mathrm{nm}$ were grown. The SEM image in Fig.17 shows that only one Ge nanowire is grown on each $\mathrm{Au}$ dot. The Au catalysts initiate and guide the growth of Ge nanowires. And the wires are located precisely where the Au catalysts sit, which may offer the possibility of in situ fabrication of large-scale nanowire devices.

\section{Conclusion}

In conclusion, the radial growth of Ge nanowire, the influence of surface condition on the growth of Ge nanowire, the growth of Ge-Si nanowire heterostructures and the patterned Ge nanowire were discussed in this chapter. The VSS growth mechanism dominates the nanowire growth in the radial direction and contributes to the increase of the diameter of nanowires. Due to the Au migration on the surface, the Au-Ge alloys on the nanowire 
surface are in solid state at the growth temperature. And this VSS mechanism contributes to the radial growth. Once the Au catalysts on the tip are consumed, the nanowires with the rough surface will be grown because of the selective-area VSS radial growth.

High-density $\mathrm{Ge}$ nanowires can be easily grown on $\mathrm{SiO}_{2}$-terminated $\mathrm{Si}$ (100) substrate. However, the $\mathrm{SiO}_{2}$ overlayer formed on the surface of Au catalysts evaporated on $\mathrm{H}$ terminated $\mathrm{Si}$ substrate prevents the growth of Ge nanowires. After removed this $\mathrm{SiO}_{2}$ overlayer by HF solution, high-density and well-ordered Ge nanowire can be obtained. Nanowires can be grown orderly along the [101], [011], [-101] and [0-11] directions.

The Au particles on the nanowire surfaces due to the surface migration play an important role in the controlling the growth of nanowire heterosturcture. Different topographies of heterostructure were observed. On the Ge nanowire stems, many Si nanowire branches were observed. Their distribution agrees well with that of Au particles on the Ge nanowire surface. However, the high-density Ge nanodots instead of branches prefer to grow on the Si nanowire stems.

Ge nanowires can be grown selectively on the area with Au catalysts. Single Ge nanowire array with a space distance of $700 \mathrm{~nm}$ was grown by LPCVD method on the patterned $\mathrm{Au}$ catalyst substrate.

\section{Acknowledgement}

This work is partially supported by SORST-JST (Japan Science and Technology) and Japan society for the promotion of science (JSPS).

\section{References}

Adhikari, H.; Marshall, A.F.; Chidsey, C. E. D.; McIntyre,P. C. (2006). Germanium nanowire epitaxy: Shape and orientation control. Nano lett. Vol. 6, pp.318-323.

Allen, J. E.; Hemesath, E. R.; Perea, D. E.; Lensch-falk, J. L.; Li, Z.Y.; Lin, F.; Gass, M. H.; Wang, P.; Bleloch, A. L.; Palmer, R. E.; Lauhon, L. J.(2008). High-resolution detection of Au catalyst atoms in Si nanowires. Nature Nanotechnol.,Vol.3, pp.168173.

Ben-Ishai, M.; Patolsky, F. (2010). A Route to High-Quality Crystalline Coaxial Core/ Multishell Ge@Si(GeSi)n and Si@(GeSi)n Nanowire Heterostructures, Adv. Mater. Vol.22,902-906.

Bierman, M. J.; Jin, S.; (2009). Potential Applications of Hierarchical Branching Nanowires in Solar Energy Conversion, Energy Environ. Sci., Vol. 2, pp.1050-1059.

Buffat, P.; Borel, J-P. (1976). Size effect on the melting temperature of gold particles. Phys. Rev. A Vol.13, pp. 2287-2298

Chan, C. K.; Peng, H.; Liu, G.; McIlwrath, K.; Zhang, X. F.; Huggins, R. A.; Cui, Y. (2008a). High-performance lithium battery anodes using Si nanowires. Nature Nanotech., Vol. 3, pp. 31-35.

Chan, C. K.; Zhang, X. F.; Cui, Y. (2008b) High Capacity Li Ion Battery Anodes Using Ge Nanowires. Nano Lett., Vol. 8, pp.307-309.

Clark, T. E.; Nimmatoori, P.; Lew, K.; Pan, L.; Redwing, J. M.; Dickey, E. C. (2008). Diameter Dependent Growth Rate and Interfacial Abruptness in Vapor-Liquid-Solid Si/Si1xGex Heterostructure Nanowires. Nano Lett. Vol.8, pp.1246-1252. 
Conley, J. F.; Stecker, J. L.; Ono, Y. (2005). Directed integration of ZnO nanobridge devices on a Si substrate Appl. Phys. Lett., Vol.87, p.223114.

Cui, Y.; Wei, Q.; Park, H.; Lieber C. M., (2001). Nanowire Nanosensors for Highly Sensitive and Selective Detection of Biological and Chemical Species.Science, Vol.293, pp.1289-1292.

Dick, K. A.; Deppert, K.; Karlsson, L. S.; Larsson, M. W.; Seifert, W.; Wallenberg, L. R.; Samuelson, L. (2007b).Directed growth of branched nanowire structures.MRS Bulletin, Vol.32, pp.127-133

Dick, K. A.; Kodambaka, S.; Reuter, M. C. ; Deppert, K.; Samuelson, L.; Seifert, W.; Wallenberg, L. R.; Ross, F. M. (2007a). The Morphology of Axial and Branched Nanowire Heterostructures. Nano Lett. Vol.7, pp. 1817-1822.

Doerk, G. S.; Ferralis, N.; Carraro, C.; Maboudian, R. (2008). Growth of Branching Si Nanowires Seeded by Au-Si Surface Migration. J. Mater. Chem. Vol.18, pp.53765381.

Ertekin, E.; Greaney, P. A.; Chrzan, D. C.; Sands, T. D. (2005). Equilibrium limits of coherency in strained nanowire heterostructures. J. Appl. Phys. Vol. 97, p.114325.

Fan, H. J.; Werner, P.; Zacharias, M. (2006).Semiconductor Nanowires: From SelfOrganization to Patterned Growth. Small, Vol. 2, pp.700-717.

Fuhrmann, B.; Leipner, H. S.; Hoche, H. R.; Schubert, L.; Werner, P.; Gosele, U.(2005). Ordered Arrays of Si Nanowires Produced by Nanosphere Lithography and Molecular Beam Epitaxy. Nano Lett., Vol.5, pp.2524-27.

Gosele U. (2006). How clean is too clean? Nature, Vol. 440, pp.34-35.

Greytak,A. B.; Lauhon,L. J.; Gudiksen M. S.; and Lieber,C. M. (2004) Growth and transport properties of complementary germanium nanowire field-effect transistors. Appl. Phys. Lett., Vol. 84, pp.4176-78.

Gudiksen, M. S.; Lauhon, L. J.; Wang, J.; Smith, D. C.; Lieber, C. M. (2002). Growth of nanowire superlattice structures for nanoscale photonics and electronics. Nature Vol. 415, pp. 617-620.

Hannon, J. B.; Kodambaka1, S.; Ross, F. M.; Tromp, R. M.(2006). The influence of the surface migration of gold on the growth of Si nanowires. Nature, Vol. 440, pp. 69-71.

He, J. H.; Hsu, J. H.; Wang, C. W.; Lin, H. N.; Chen, L. J.; Zhong, L.; Wang, Z. L.2006. Pattern and Feature Designed Growth of ZnO Nanowire Arrays for Vertical Devices. J. Phys. Chem. B, Vol.110, pp.50-53.

Hiraki, A.; E. Lugujjo, Nicolet M.-A.; Mayer, J. W. (1971). Low-temperature migration of Si through metal films importance of Si-metal interface.Physica Status Solidi a, Vol.7, pp.401-406.

Hiraki, A. (1984).Low temperature reactions at Si/metal interfaces; What is going on at the interfaces? Surf. Sci. Rep., Vol.3, pp. 357-412.

Holmes, J. D.; Johnston, K. P.; Doty, R. C.; Korgel, B. A. (2000). Control of Thickness and Orientation of Solution-Grown Si Nanowires. Science, Vol. 287, pp.1471-1473.

Hu, Y. J.; Churchill, H. O. H.; Reilly, D. J.; Xiang, J.; Lieber, C. M.; Marcus, C. M. (2007). A $\mathrm{Ge} / \mathrm{Si}$ heterostructure nanowire-based double quantum dot with integrated charge sensorNature Nanotechnol., Vol.2, pp. 622-625.

Hu, Y.; Xiang, J. ; Liang, G.; Yan, H.; Lieber, C. M. (2008). Sub-100 Nanometer Channel Length Ge/Si Nanowire Transistors with Potential for $2 \mathrm{THz}$ Switching Speed. Nano Lett. Vol.8, pp. 925-930. 
Jagannathana, H.; Nishi, Y.; Reuter,M.; Copel, M.; Tutuc, E.; Guhab, S.; Pezzia, R. P. (2006). Effect of oxide overlayer formation on the growth of gold catalyzed epitaxial $\mathrm{Si}$ nanowire. Appl. Phys. Lett., Vol. 88, p.03113.

Kastnery, G.; Gosele, U. (2004). Stress and dislocations at cross-sectional heterojunctions in a cylindrical nanowire. [Quick Edit]Philos. Mag. Vol.84, pp.3803-3824.

Kamins, T. I.; Li, X.; Williams, R. S.; Liu, X. (2004). Growth and Structure of Chemically Vapor Deposited Ge Nanowires on Si Substrates. Nano Lett., Vol. 4, 503-506.

Kodambaka, S.; Tersoff, J.; Reuter, M. C.; Ross, F. M.(2007). Germanium Nanowire Growth Below the Eutectic Temperature.Science, Vol. 316, pp.729-732.

Lauhon, L. J.; Gudiksen, M. S.; Wang D.; and Lieber, C. M. (2002). Epitaxial core-shell and core- multishell nanowire heterostructures. Nature, Vol. 420, pp. 57-61.

Lay G. Le (1983).Physics and electronics of the noble-metal/elemental-semiconductor interface formation: A status report. Surf. Sci. , Vol.132, pp.169-204.

Li, C. B.; Cheng, B.W.; Mao, R.W.; Zuo, Y.H.; Shi , W.H.; Huang, C. J.; Luo, L.P.; Yu, J.Z.; Wang, Q.M. (2004b). Novel room temperature photoluminescence of Ge/Si islands in multilayer structure grown on Si-on-insulator substrate, Thin Solid Films, Vol.467, pp.197-200.

Li, C. B.; Mao, R. W.; Zuo, Y. H.; Zhao, L.; Shi, W. H.; Luo, L. P.; Cheng, B. W.; Yu, J. Z.; Wang, Q. M. (2004a). $1.55 \mathrm{~mm}$ Ge islands resonant-cavity-enhanced detector with high-reflectivity bottom mirror, Appl. Phys. Lett. Vol.85, pp. 2697-2699.

Li, C. B. ; Usami, K.; Mizuta, H.; Oda. S. (2009a). Vapor-solid-solid radial growth of Ge nanowires. J. Appl. Phys. Vol.106, P.046102

Li,C. B.; Usami, K.; Muraki, T.; Mizuta, H.; and Oda S., (2008). The impacts of surface conditions on the vapor-liquid-solid growth of germanium nanowires on Si (100) substrate. Appl. Phys. Lett. Vol. 93, p. 041917.

Li, C. B.; Usami, K.; Mizuta, H.; Oda, S. (2011).Growth of Ge-Si nanowires heterostructure via chemical vapour deposition, Thin solid films, doi:10.1016/j.tsf.2011.02.005.

Li, C. B.; Usami, K.; Yamahata, G.; Tsuchiya, Y.; Mizuta, H.; Oda, S. (2009b).Positioncontrollable Ge Nanowires growth on Patterned Au Catalyst Substrate, Applied Physics Express, Vol.2, p.015004

Li, J.Y.; Lu, C. G.; Maynor, B.; Huang, S. M.; Liu, J. (2004c). Controlled growth of long GaN nanowires from catalyst patterns fabricated by "dip-pen" nanolithographic techniques, Chem. Mater., Vol. 16, pp.1633-1636.

Li, S.; Liu, N. Y.; Chan-Park, M. B.; Yan, Y. H. and Zhang Q. (2007). Aligned single-walled carbon nanotube patterns with nanoscale width, micron-scale length and controllable pitch. Nanotechnology, Vol.18, p.455302.

Liang, G. C.; Xiang, J.; Kharche, N.; Klimeck, G.; Charles, M.; Lieber, C. M.; Lundstrom, M.(2007). Performance Analysis of a Ge/Si Core/Shell Nanowire Field-Effect Transistor.Nano Lett., Vol. 7, pp. 642-646.

Lombardi, I.; Hochbaum, A. I.; Yang, P. D.; Carraro, C.; Maboudian, R. (2006), Synthesis of high density, size-controlled Si nanowire arrays via porous anodic alumina mask, Chem. Mater., Vol.18, 988-991.

Lu, W.; Xiang, J.; Timko, B. P.; Wu, Y.; Lieber, C. M. (2005). One-dimensional hole gas in germanium/Si nanowire heterostructures, Proc. Natl. Acad. Sci. Vol.102, pp.10046. 
Sun, X. H.; Calebotta, G.; Yu, B.; Selvaduray G.; Meyyappan, M.;(2007). Synthesis of germanium nanowires-on-insulator (GeNOI) catalyzed by indium or antimony. $J$. Vac. Sci. Technol. B, Vol. 25, 415-420.

Tyryshkin, A. M.; Lyon, S. A.; Schenkel, T.; Bokor, J. ; Chu, J.; Jantsch, W.; Schaffler, F. ; Truitt, J. L.; Coppersmith S. N.; Eriksson, M. A.. (2006). Electron spin coherence in Si,Physica E, Vol. 35, 257-263

Venables, J. A., (1986). Nucleation and growth process in thin film formation, J. Vac. Sci. Technol. B, Vol.4, pp. 870-873.

Wang, D.; Qian, F.; Yang, C.; Zhong, Z.; Lieber, C. M. (2004). Rational Growth of Branched and Hyperbranched Nanowire Structures. Nano Lett. Vol.4, pp. 871-874.

Wu, Y. Y.; Fan, R.; Yang, P. D. (2002). Block-by-Block Growth of Single-Crystalline Si/SiGe Superlattice Nanowires.Nano Lett. Vol.2, pp.83-86.

Xiang, J.; Lu, W.; Hu, Y. J.; Wu, Y.; Yan, H.; Lieber, C. M. (2006). Ge/Si nanowire heterostructures as high performance field-effect transistors. Nature, Vol.441, pp. 489-493.

Y. Maeda, N. Tsukamoto, Y. Yazawa, Y. Kanemitsu and Y. Masumoto, (1991). Visible photoluminescence of Ge microcrystals embedded in $\mathrm{SiO}_{2}$ glassy matrices. Appl. Phys. Lett., Vol. 59, pp. 3168-3170 


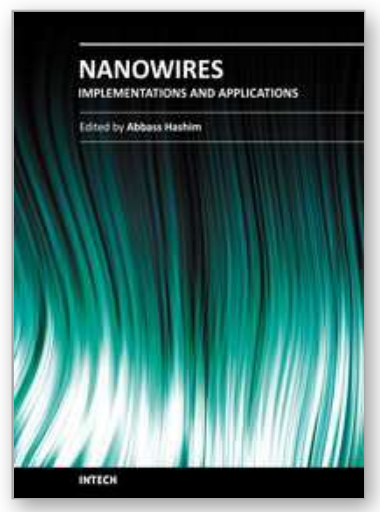

\author{
Nanowires - Implementations and Applications \\ Edited by Dr. Abbass Hashim
}

ISBN 978-953-307-318-7

Hard cover, 538 pages

Publisher InTech

Published online 18, July, 2011

Published in print edition July, 2011

This potentially unique work offers various approaches on the implementation of nanowires. As it is widely known, nanotechnology presents the control of matter at the nanoscale and nanodimensions within few nanometers, whereas this exclusive phenomenon enables us to determine novel applications. This book presents an overview of recent and current nanowire application and implementation research worldwide. We examine methods of nanowire synthesis, types of materials used, and applications associated with nanowire research. Wide surveys of global activities in nanowire research are presented, as well.

\title{
How to reference
}

In order to correctly reference this scholarly work, feel free to copy and paste the following:

Chuanbo Li, Hiroshi Mizuta and Shunri Oda (2011). Growth and characterisation of Ge Nanowires by chemical vapour deposition, Nanowires - Implementations and Applications, Dr. Abbass Hashim (Ed.), ISBN: 978-953307-318-7, InTech, Available from: http://www.intechopen.com/books/nanowires-implementations-andapplications/growth-and-characterisation-of-ge-nanowires-by-chemical-vapour-deposition

\section{INTECH}

open science | open minds

\section{InTech Europe}

University Campus STeP Ri

Slavka Krautzeka 83/A

51000 Rijeka, Croatia

Phone: +385 (51) 770447

Fax: +385 (51) 686166

www.intechopen.com

\section{InTech China}

Unit 405, Office Block, Hotel Equatorial Shanghai

No.65, Yan An Road (West), Shanghai, 200040, China

中国上海市延安西路65号上海国际贵都大饭店办公楼 405 单元

Phone: +86-21-62489820

Fax: +86-21-62489821 
(C) 2011 The Author(s). Licensee IntechOpen. This chapter is distributed under the terms of the Creative Commons Attribution-NonCommercialShareAlike-3.0 License, which permits use, distribution and reproduction for non-commercial purposes, provided the original is properly cited and derivative works building on this content are distributed under the same license. 